\title{
Transformer DC Magnetic Bias Phenomena in HVDC
}

\author{
Lijun Qin ${ }^{1, a}$, Wantao Yang ${ }^{1, a}$ \\ ${ }^{1}$ Institute of Electrical and Electronic Engineering, North China Electric Power University, Beijing, \\ 102206, China \\ aemail:786331013@qq.com
}

Keywords: HVDC; DC magnetic bias; Transformer

\begin{abstract}
The ground current would lead to transformer DC magnetic bias in HVDC unipolar runtime, causing the transformer noise increases, increased vibration, overheating and other hazards, adverse affect on the series capacitors and even the grid. It introduces the research background and significance, the mechanism of DC magnetic bias, harm and suppression measures.
\end{abstract}

\section{Introduction}

HVDC is currently widely used in Chinese national network, the west to east and the north-south trend of electricity supply due to a large-capacity long-distance transmission capacity, low transmission losses and save overhead line corridors, etc. .

But there are some problems about HVDC, such as the widely used for HVDC converter stations both ends of a bipolar neutral grounding mode. ground zero Loop current during normal operation, but once the operation current of the poles are not equal (such as the use of unipolar run), there were current flows between grounding poles, resulting in DC magnetic bias. This will cause the voltage waveform distortion, following the malfunction of security device , transformer losses increase, increased vibration, noise increases and so on.

UHV transformer is an important equipment of HVDC. If DC magnetic bias cause the protective device of UHV transformer malfunction, it would seriously endanger the safe operation of UHV power grid. Therefore, for the further development of HVDC, studying the effects and the mechanism of DC magnetic bias, and proposing effective suppression measures, has important implications for the transformer to improve efficiency, extend the life and improve the harmonic environment.

\section{Magnetic Bias Mechanism Analysis}

DC magnetic bias refers to the transformer magnetizing current occurs the DC component, leading to the transformer core a half weeks of magnetic saturation, and thus triggered a series of effects.

The relationship $\phi=f(i)$ between flux in the transformer core $\phi$ and the current in magnetizing coil $i$ is non-linear. As shown in Fig.1(b) shows, for the maximum efficiency, the transformer rated operating point is A,which is the endpoint of the linear part in Fig.1(b). In normal operation, magnetizing coils superimposed to DC current can make excitation current curve up or down shift along the coordinate axis . AC and DC magnetic flux superimposed on each other. If the flux enter the magnetization curve saturated zone, the excitation current will operate in the saturation region. Then waveform distortion occurs, creating top wave, as shown in Figure 1 (c).

There are mainly two ways to produce DC magnetic bias current : The First is geomagnetic induced currents (GIC) due to solar activity, the second is HVDC a single-pole- the land run.

\section{"Geomagnetic Storm" Generated by the Interaction of Solar Plasma Wind Dynamic Changes and the Geomagnetic Field}

Solar activity, especially in the flares, high-temperature plasma fly to hit the Earth (hundreds of kilometers per second), despite the Earth's magnetosphere blocking, there is still a small part of the 
plasma flow into the near-Earth region, which makes the geomagnetic field dramatic changes in short time. The surface occurs potential gradient . Serious, ground potential gradient in some areas will reach a few volts per kilometer, or even hundreds volts, a few minutes to a few hours. When the electric field is applied to the neutral grounding transformer, the transformer windings will produce GIC, its frequency lower (0.001 IHz), approximately DC, sizes up to $80 \sim 100$ A or more.

\section{System Unipole - Earth Run}

When the same area run the AC and DC systems at the same time, especially runing under HVDC system unipolar-earth loop mode, if there is neutral grounding transformers, due to soil resistance radial distribution uneven, and underground may have a metal pipe or a metal such as armored cable, which provides current a more excellent conductive path than the ground soil , a part of the current will flow along through these metal distance, resulting in that it is difficult to determine respective substation neutral point elevation potential. This will inevitably lead to the existence of a certain potential difference between the various ground. Such a potential difference will cause a certain DC current from the primary side of the transformer neutral point inject into the transformer.

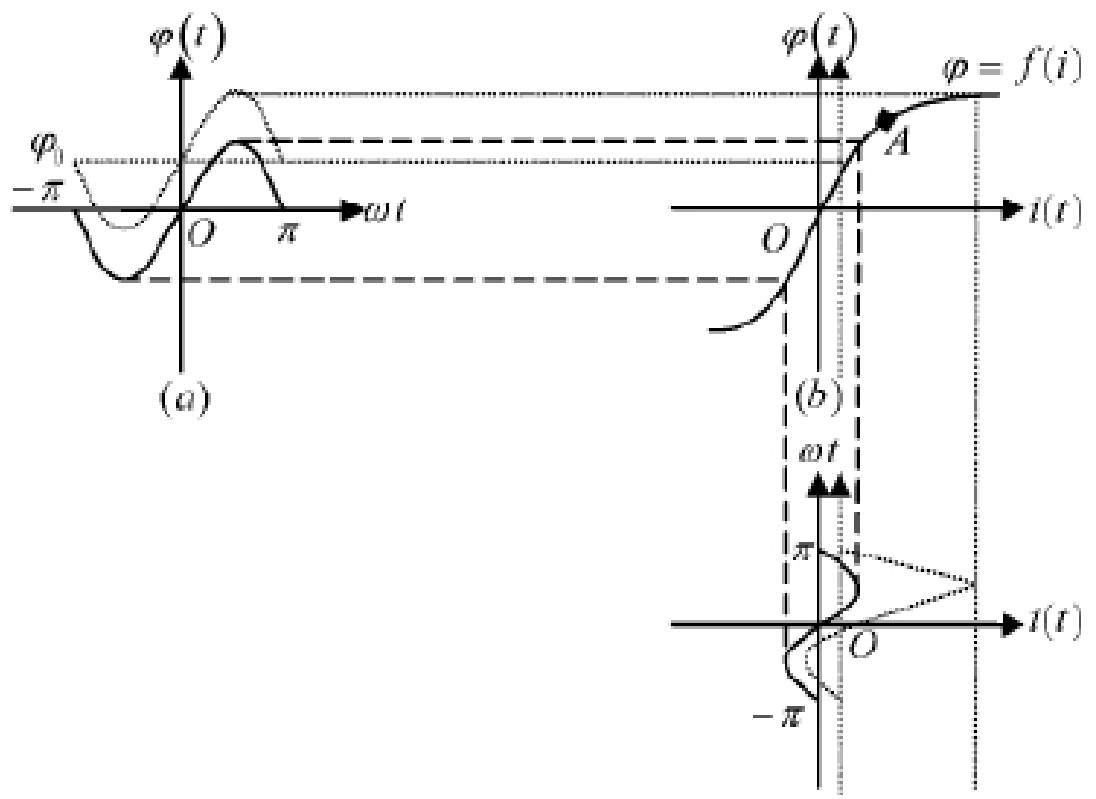

(c)

Fig.1.DC current impacts transformer magnetizing current

\section{Magnetic Bias Effects and Hazards}

When DC magnetic bias occurs, DC flux superimposes on the AC excitation flux to synthesis total flux density, resulting half cycle of the total flux density in the same direction with the DC magnetic bias is greatly increased, while the other half of the cycle is reduced, leading to excitation current waveform rendering spire waves.

\section{Impact to the Transformer}

DC magnetic bias's main hazard is the impact on the transformer, the following main points:

1, the transformer magnetizing current distortion. 2, the impact of the transformer waveform. If the core work in a serious saturation area, increased leakage flux in a certain extent flatten the voltage peak . 3, increasing the transformer losses. Transformer magnetizing current may be a substantial increase in the role of DC current, and the transformer basic copper consumption increased dramatically. 4, resulting in transformer vibration increased. DC magnetic bias change the operating point of the original transformer, making part of the original core work area to move to the saturated zone, corresponding to the total excitation current becoming steeple wave, causing the transformer vibration increases. 5, the noise increases.

\section{The Impact on the Shunt Capacitor}

DC magnetic bias will produce a lot of harmonic, increasing the total harmonic distortion, 
causing substation low-voltage side over-current, causing the capacitor explosion. A Chinese $500 \mathrm{kV}$ substation runs in HVDC monopolar ground loop mode. Its capacitor banks at side of the low-pressure occurred explosion many times, monitoring the harmonic currents up to $200 \mathrm{~A}$ and above.

\section{Hazards on the Grid}

DC magnetic bias will cause voltage drop, following the security device malfunction and other hazards.Canada Quebec blackout is the most severely power outages affected by DC magnetic bias, causing the power interruption up to nine hours in the area of the accident, during which time nearly 600 million people have no electricity, which has brought about a huge impact and economic losses.

\section{Magnetic Bias Suppression Measures}

\section{Neutral Series Resistance}

Series resistance equivalently increases ground branch resistance, there will be more current flows through the earth branches, thereby limiting the ground current going into AC system . Resistance selection should consider the size of the curbing DC current and the neutral point over-voltage and other aspects , too big or too small may result in adverse outcomes.

\section{Series Capacitor}

The neutral connecting series capacitor to the ground take use of capacitor 'no DC but AC' features to suppress DC current. Normally capacitor isolates DC current, as well as provides access to AC. When a fault occurs, the neutral will flow through the larger current, resulting in a higher amplitude transient voltage. Once the capacitor voltage exceeds the critical value, the current protection device will bypass capacitor to suppress the transient voltage. After troubleshooting, the capacitor is back into run.

\section{Reverse Injection Current}

Also known as Potential Compensation Act,which use reverse DC current to attenuate the adverse effects of the original DC current. The method do not need to change the system parameters, and does not affect insulation coordination, automatic device, protection, etc.. The structure is simple, economic and easy.

\section{Summary}

This article describes DC magnetic bias research technical background, the reason, harm and suppression measures. In view of the uncertainty of HVDC system grounding electrode current and geomagnetic induced current distribution and Chinese transformer DC magnetic bias is no clear standards for affordability. Transformer DC magnetic bias is still a major problem faced by the power system, which need us strive to resolve.

\section{References}

[1] Haiqing Lu. Impact of HVDC transmission ground running on AC power grid unipolar research [D] Zhejiang: Zhejiang University, 2013.

[2] Yongliang Hou.research process HVDC transformer DC bias phenomenon [D] Hebei: North China Electric Power University (Baoding), 2006.

[3] Wei Jiang.UHVDC transmission causes transformer DC bias and suppression measures [D] Sichuan: Southwest Jiaotong University, 2009.

[4] Jyh-Wei Lee, Shih-Kang Tien, Yu-Chu Kuo. The effects of pulse frequency and substrate bias to the mechanical properties of CrN coatings deposited by pulsed DC magnetron sputtering[J].Thin Solid Films, 2005, 494(1).

[5] Feng Du, Ying Zhang. DC bias effect on the transformer and suppression measures [J]. Transformer. 2008 (07) 